\title{
Latitudinal distribution of earthquakes in the Andes and its peculiarity
}

\author{
B. W. Levin ${ }^{1}$ and E. V. Sasorova ${ }^{2}$ \\ ${ }^{1}$ Institute of Marine Geology and Geophysics, Far East Branch of Russian Academy of Sciences, Yuzhno-Sakhalinsk, Russia \\ ${ }^{2}$ Shirshov Institute of Oceanology of Russian Academy of Sciences, Moscow, Russia
}

Received: 25 March 2009 - Revised: 31 August 2009 - Accepted: 4 September 2009 - Published: 14 December 2009

\begin{abstract}
In the last decade, there has been growing interest in problems related to searching global spatiotemporal regularities in the distribution of seismic events on the Earth. The worldwide catalogs ISC were used for search of spatial and temporal distribution of earthquakes (EQ) in the Pacific part of South America. We extracted all EQ from 1964 to 2004 with $\mathrm{Mb}>=4.0$. The total number of events under study is near 30000 . The entire set of events was divided into six magnitude ranges (MR): $4.0<=\mathrm{Mb}<4.5 ; \quad 4.5<=\mathrm{Mb}<5.0 ; \quad 5.0<=\mathrm{Mb}<5.5$; $5.5<=\mathrm{Mb}<6.0 ; \quad 6.0<=\mathrm{Mb}<6.5 ;$ and $6.5<=\mathrm{Mb}$. Further analysis was performed separately for each MR. The latitude distributions of the EQ number for all MR were studied. The whole region was divided in several latitudinal intervals (size of each interval was either $5^{\circ}$ or $10^{\circ}$ ). The number of events in each latitudinal interval was normalized two times. After normalization we obtained the relative seismic event number generated per one kilometer of plate boundary. The maximum of seismic activity in the Pacific part of the South America is situated in latitude interval $20^{\circ}-30^{\circ} \mathrm{S}$. The comparative analysis was executed for the latitude distributions of the EQ number and the EQ energy released. Then the distributions of EQ hypocenter location in latitude and in depth were studied. The EQ sources for the high latitudes (up to $35^{\circ} \mathrm{S}$ ) are located on the depth (H) between $20-80 \mathrm{~km}$. It was shown, that full interval of depth in each latitudinal belt generally divides into three parts (clusters) with closecut separation boundaries $(\mathcal{K} 1-$ with $0<\mathrm{H}<=80 \mathrm{~km}, \mathcal{K} 2-$ with $120<\mathrm{H}<=240 \mathrm{~km}$ and $\mathcal{K} 3-$ with $\mathrm{H}>=500 \mathrm{~km}$ ).
\end{abstract}

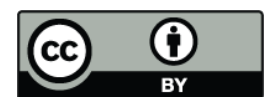

Correspondence to: $\mathrm{B}$. W. Levin (levinbw@mail.ru)

\section{Introduction}

The search for global regularities in the latitudinal distribution of earthquakes even in the epoch of the formation of seismological science demonstrated a clear inhomogeneity in the distribution of epicenters over the Earth despite low representativeness of the observational material in the middle of the 20th century (Gutenberg, Richter, 1954).

Quantitative estimates of earthquake distribution by latitudinal zones of the planet based on electronic catalogues were obtained firstly by Mogi (1985), then (Sun, 1992; Levin and Chirkov, 2001). It was shown that the seismic activity of the planet, which is almost absent at the poles and polar caps of the Earth, increases significantly at mid-latitudes reaching the maximum in the region of $40^{\circ}-50^{\circ} \mathrm{N}$ and $10^{\circ}-20^{\circ} \mathrm{S}$ with a stable local minimum near the equator.

Previous attempts to normalize the number of events by the square of the latitudinal zone were ineffective and physically wrong due to a strong irregularity in the distribution of earthquakes within the latitudinal zones. Taking into account the fact that the earthquakes occur generally along the boundaries of the lithosphere plates and that the modern kinematics of the plate boundaries is studied quite well was applied normalizing the number of events in the latitudinal zone by the total length of the plate boundaries in the given zone (Levin and Sasorova, 2009). Thus the average number of seismic events generated per one kilometer of plate boundary for each latitudinal belt was obtained. Double normalized (binormed) latitudinal distributions of the EQ number (Fig. 1) have clearly expressed bimodal character with two peaks located in Northern Hemisphere $\left(40^{\circ}-50^{\circ} \mathrm{N}\right.$, i.e. Kuril Islands and Japan) and in Southern Hemisphere $\left(20^{\circ}-30^{\circ} \mathrm{S}\right.$, i.e. Oceania northward to New Zealand and South America), local minimum near the equator $\left(10^{\circ}-20^{\circ} \mathrm{N}\right)$ and almost zero values in the regions of the polar caps. 


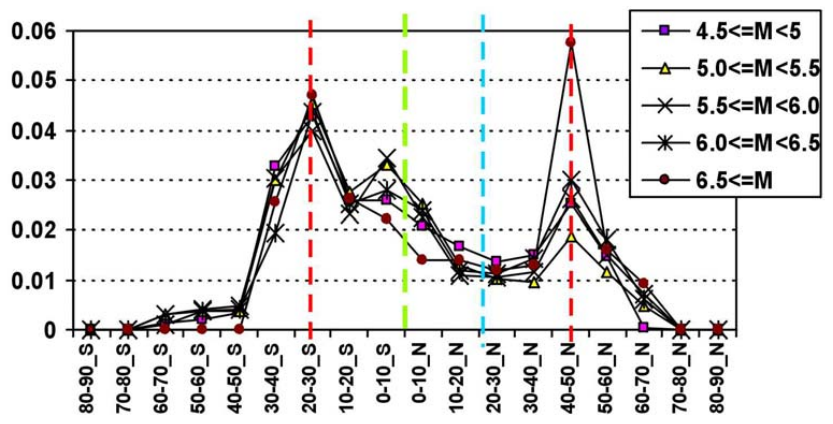

Fig. 1. Binormed latitudinal distributions of earthquakes in the $\mathrm{Pa}-$ cific region for five magnitude ranges; the horizontal axis is the latitudinal belts; vertical axis is binormed number of earthquakes. Two red dotted lines correspond to peaks of binormed distribution; blue dotted lines correspond to local minimum and green dotted line is situated on equator.

\section{Data preprocessing}

The objective of this work is the analysis as the latitude-depth distributions of the EQ number and the latitude-depth distributions of the EQ energy released for the Pacific part of the South America. The Pacific part of South America is defined as the ocean basin, and land regions over subduction zones. It was analyzed that number of earthquakes in the spreading zones in the South Pacific does not exceed 2\% of the total number of events in each latitudinal zone. Thus these events were canceled from list of events.

In order to analyze spatial distributions of earthquakes, we used data from the World Electronic Catalog of the International Seismic Catalog (hereafter, ISC catalog) [ISC] from 1964 to 2004 with magnitude $\mathrm{Mb}>=4$. The preliminary standardization of magnitudes (to Mb value) was fulfilled for all events. Aftershocks were canceled from the list of events for study of latitudinal distributions of the EQ number (using the program by V. B. Smirnov, Moscow State University). The total number of events was near 30000 . The entire set of events under analysis was divided into six magnitude ranges (MR): $4.0 \leq \mathrm{Mb}<4.5 ; 4.5 \leq \mathrm{Mb}<5.0 ; 5.0 \leq \mathrm{Mb}<5.5$; $5.5 \leq \mathrm{Mb}<6.0 ; 6.0 \leq \mathrm{Mb}<6.5$; and $6.5 \leq \mathrm{Mb}$. The map of the South America with events under study is presented on the Fig. 2a.

The Gutenberg-Richter power-law relationship for seismic events in studied part of South America is presented on the Fig. 2b. The angle of bend in upper part of the curve conforms to $\mathrm{Mb}$ value equal to 4.3. Thus the magnitude range $4.0<=\mathrm{Mb}<4.5$ can not be considered as completeness and it was eliminated. Therefore, we compiled earthquake subsets for each studied segment with respect to five MRs (without the MR $4.0<=\mathrm{Mb}<4.5$ ). Further analysis was performed separately for each chosen MR. The separation of event set into several subsets with different magnitude ranges allows us to analyze the peculiarity of the seismic process for var- ious energy levels. In upper-right corner of the Fig. $2 b$ is situated the equation of linear regression and the $R^{2}$ value (unbiased estimate of determination factor). Hence residual dispersion, which determined by random component, is very small.

The latitude distributions of the EQ number for all magnitude ranges were studied. The region which is under study was divided in several latitudinal intervals (size of each interval was either $10^{\circ}$ or $5^{\circ}$ ). The number of events in each latitudinal interval was normalized two times. At first number of events in each latitudinal belt was divided on total number of events in given MR. Then second normalization was carried out with respect to the length of lithosphere plate boundaries in the given latitudinal zone. Thus we obtain average relative number of seismic event generated per one kilometer of plate boundary.

\section{Analysis}

Firstly the latitudinal distributions of the EQ number for five MRs with size of each latitudinal belt equal to $10^{\circ}$ were discussed. The binormed EQ distributions in latitude for five MR are presented on the Fig. 3. It may be marked one clearly expressed peak in latitudinal belt $20^{\circ}-30^{\circ} \mathrm{S}$ for the all MRs and almost zero values of the EQ number in the high latitudes $\left(90^{\circ}-60^{\circ} \mathrm{S}\right)$. The same peak and the negligible small EQ number in the high latitudes were detected for the latitudinal distributions in Southern Hemisphere for whole Pacific (Fig. 1).

The problem of time stability of presented distributions is one of the debatable problems in the works on global seismicity. In discussed above case the full interval of observation was forty years. Then, we specially analyzed the latitudinal distributions over four 10 -year time intervals. The latitudinal distributions of binormed number of the EQ in four ten-year periods for MR: $4.5<=\mathrm{Mb}<5,5<=\mathrm{Mb}<5.5$, and $5.5<=\mathrm{Mb}<6$ are shown on Fig. $4 \mathrm{a}, \mathrm{b}$, and c, respectively. The distributions of relative number of the EQ for all MR together in four ten-year periods are shown on the Fig. $4 \mathrm{~d}$.

It may be observed, that the latitudinal distributions in different ten-year periods vary practically insignificant for all MR. Sometimes peak shifted for some MR from $\left(20^{\circ}-30^{\circ} \mathrm{S}\right)$ to $\left(30^{\circ}-40^{\circ} \mathrm{S}\right)$. Thus character of distributions is generally conserved in all time intervals considered. The most pronounced stability is found for the latitudinal distributions of the relative number of the EQ for all MR (Fig. 4d).

Next the latitudinal distributions of the EQ number for five MRs with more detailed size of each latitudinal belt $\left(5^{\circ}\right)$ were studied. In this case we discharge the high latitudes from $55^{\circ}$ to $90^{\circ} \mathrm{S}$ from all plot of the Fig. 5 because of negligible small number of events in these latitudes. The binormed EQ distributions in latitude for five MR are presented on the Fig. 5b. The total number of events in each MR is located in the rightmost upper rectangle. 
a)

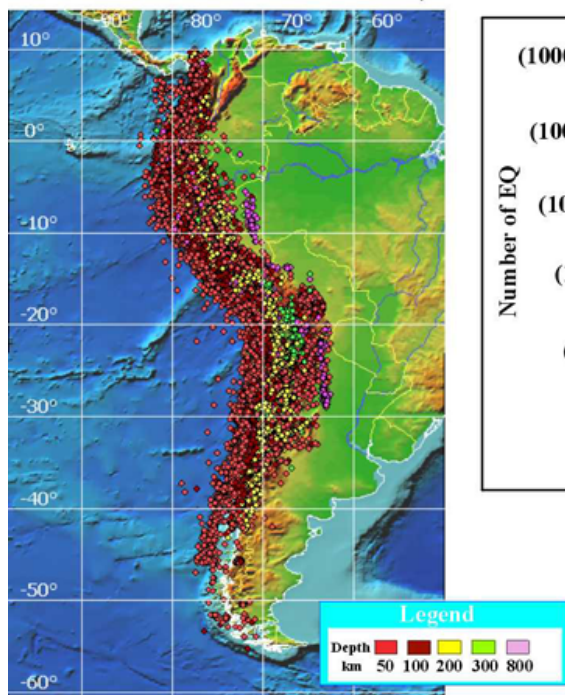

b)

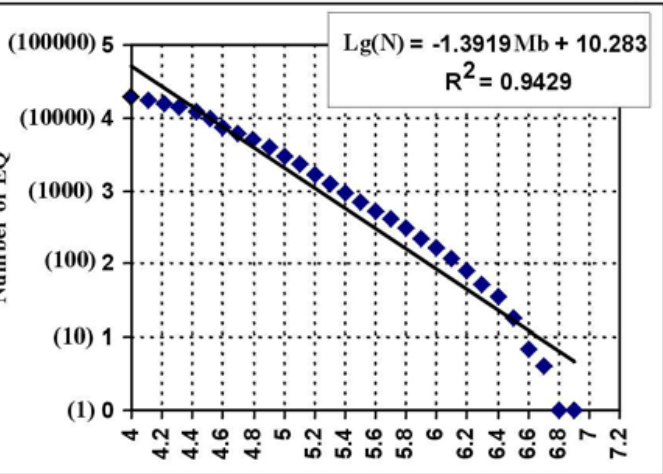

Fig. 2. (a) is the map of the South America with events under study (small circles); the color of each circles depends on source depth; (b) is the Gutenberg-Richter power-low relationship for seismic events in studied part of the South America.

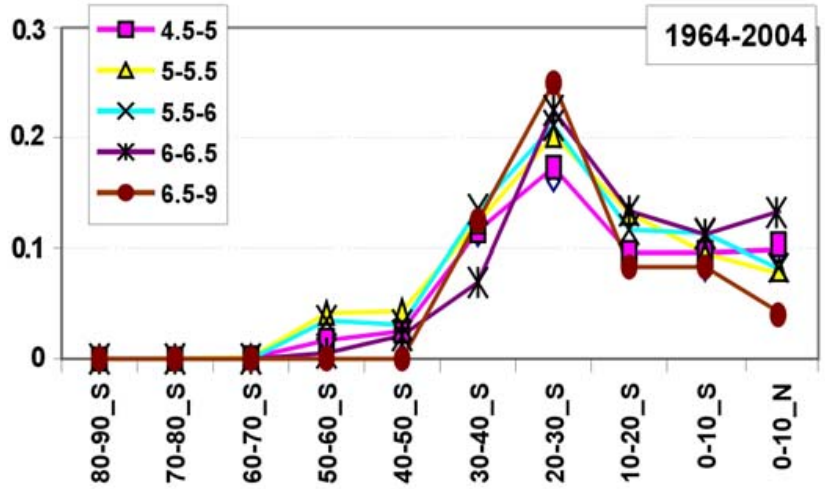

Fig. 3. Binormed latitudinal distributions of earthquakes in the Pacific part of the South America for five magnitude ranges. The horizontal axis is the latitudinal belts; vertical axis is binormed number of earthquakes.

It may be marked that in this case primary peak for $10^{\circ}$ latitudinal distribution $\left(20^{\circ}-30^{\circ} \mathrm{S}\right)$ on Fig. 3 for the most MR partitioned in two clearly expressed peaks in two latitudinal belts $\left(30^{\circ}-35^{\circ} \mathrm{S}\right.$ and $\left.20^{\circ}-25^{\circ} \mathrm{S}\right)$ and local minimum: $25^{\circ}-30^{\circ} \mathrm{S}$. The peaks were marked on by red dotted lines on Fig. 5, and by bold red arrows on the map of the Pacific part of the South America, which is presented on Fig. 5a. These are the zones of the seismic activity maximum.

The distributions of the energy released by EQs (lilac line) and the normalized energy released by EQ (dark blue line) shown on Fig. 5c. The normalization was performed by the length of lithosphere plate boundaries in each latitudinal belt. The distributions on Fig. 5c (the binormed EQ number for magnitude range $\mathrm{Mb}>=6.5$ ) and on Fig. $5 \mathrm{~d}$ are quite similar.
Then we take into consideration 3-D distributions of the EQ relative number: in latitude, in depth, and in MR. The graphs of distributions in depth for the events in each latitudinal belt are presented on Fig. 6. The vertical axes on all presented plots are relative number of the EQs in given latitudinal belt.

One can see, that up to $90 \%$ of the EQ sources in the high latitudes are located on the depth $20<=\mathrm{H}<=60 \mathrm{~km}$. The essential part of the EQ sources in latitudinal belts near equator $\left(30^{\circ} \mathrm{S}-0^{\circ}\right)$ are located on the depths $100<\mathrm{H}<=240 \mathrm{~km}$ (intermediate EQ) and $\mathrm{H}>=500 \mathrm{~km}$ (deep EQ). Then the part of shallow EQ increased up to $90 \%$ for the latitudes from equator to $10^{\circ} \mathrm{N}$.

The distribution analysis of the EQ energy released in depth and in latitude shows (Fig. 7), that full interval of depth in each latitudinal belt may be divided into several isolated parts (clusters) with close-cut separation boundaries $(\mathcal{K} 1-$ with $0<\mathrm{H}<=80 \mathrm{~km}, \mathcal{K} 2-$ with $120<\mathrm{H}<=240 \mathrm{~km}$ and $\mathcal{K} 3-$ with $\mathrm{H}>=500 \mathrm{~km}$ ).

We can see that more than $90 \%$ of energy for the latitudinal belts from $55^{\circ}-50^{\circ} \mathrm{S}$ to $40^{\circ}-35^{\circ} \mathrm{S}$ releases by the EQ which sources are located on the depth $\mathrm{H}<80 \mathrm{~km}$ (cluster $\mathcal{K} 1$ ). And the energy released by the EQs from clusters $\mathcal{K} 2$ and $\mathcal{K} 3$ is negligible for these latitudes. But the energy released by the EQs in the middle latitudinal belts $\left(35^{\circ}-15^{\circ} \mathrm{S}\right)$ partitions between clusters $\mathcal{K} 1$ and $\mathcal{K} 2$. Noticeable part of energy which released by EQs with depth $\mathrm{H}>=500 \mathrm{~km}$ (cluster $\mathcal{K} 3)$ appears in latitudes which located near equator $\left(15^{\circ}\right.$ $\left.0^{\circ} \mathrm{S}\right)$. Practically all energy for the northern latitudes $\left(0^{\circ}\right.$ $10^{\circ} \mathrm{N}$ ) releases by shallow EQs (cluster $\mathcal{K} 1$ ). It should be noted that in the latitudinal belts with peaks of seismic activities $\left(30^{\circ}-35^{\circ} \mathrm{S}\right.$ and $\left.20^{\circ}-25^{\circ} \mathrm{S}\right)$ the energy distributions 

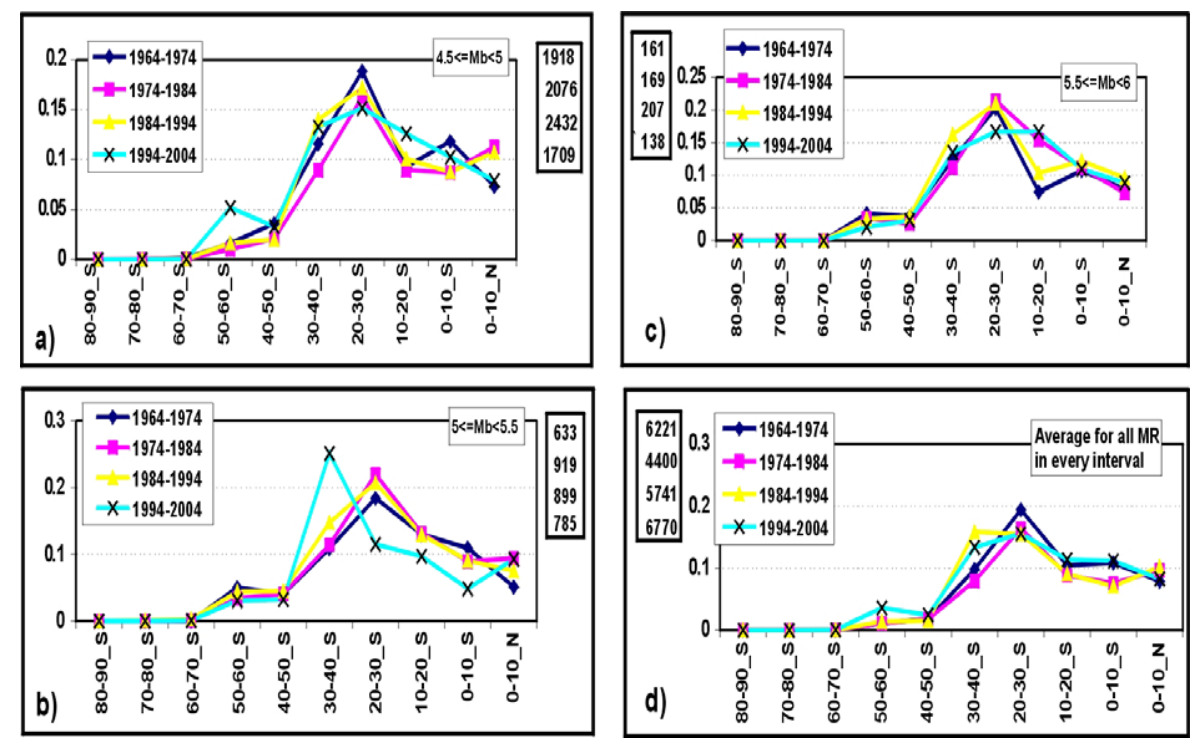

Fig. 4. Latitudinal distributions of the earthquakes in the Pacific part of the South America for four 10-year time intervals. The horizontal axes are the latitudinal belts; vertical axes are the binormed numbers of events. (a), (b), and (c) are latitudinal distributions of events for three different magnitude ranges; (d) is distribution of the mean value calculated from five magnitude ranges over four 10-year periods. The total numbers of seismic events in the corresponding time period are shown in rectangle in the upper right corner of (a) and (b) and in upper left corner of (c) and (d).

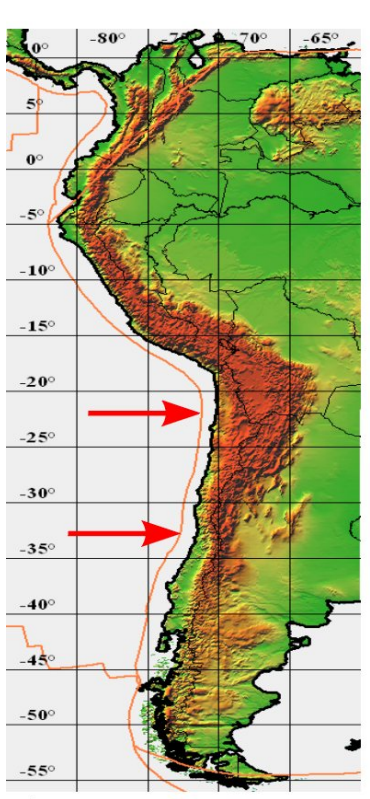

a)
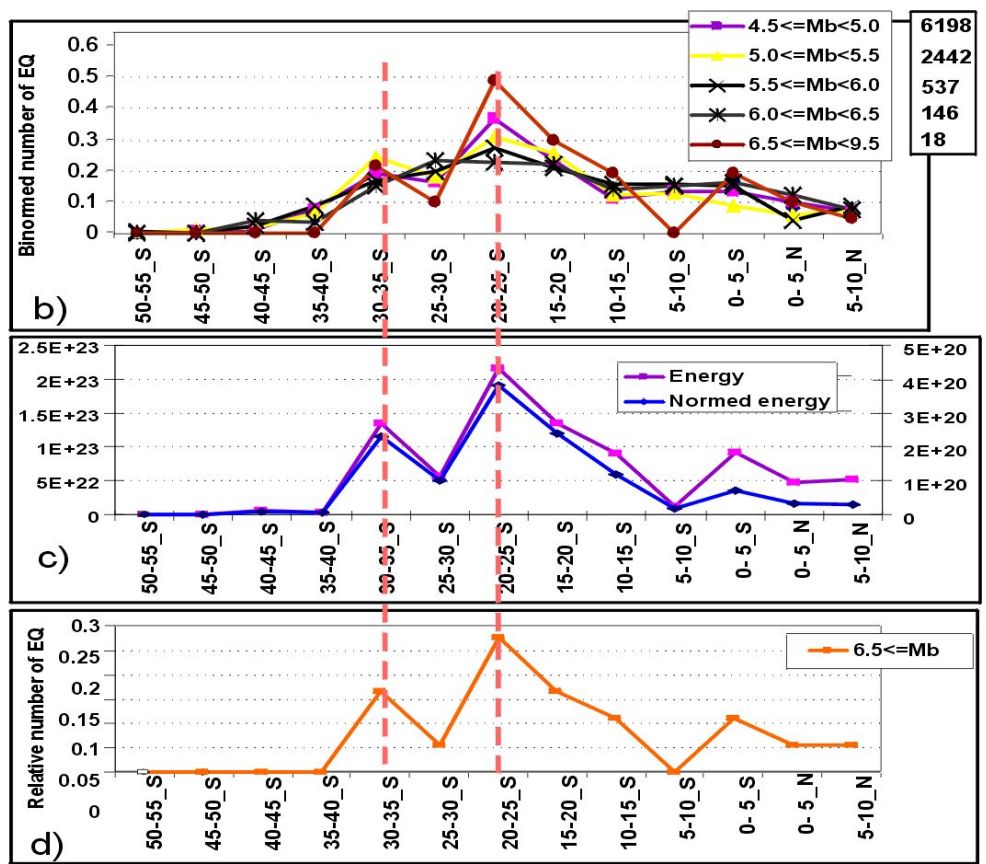

Fig. 5. (a) is the map of the South America with lithosphere plate boundaries (orange line). (b) is presented the binormed latitudinal distributions for South America (13 latitudinal belts with scale interval $5^{\circ}$ ) for five magnitude ranges; the legend for MR is situated in upper right corner. (c) is the latitudinal distributions of the released EQ energy (left vertical axis) and normalized energy of the EQ (right vertical axis) over latitudinal belts. (d) is the binormed latitudinal distributions of the EQ number for magnitude range: Mb>=6.5. Horizontal axes for all plots are the latitudinal belts. 


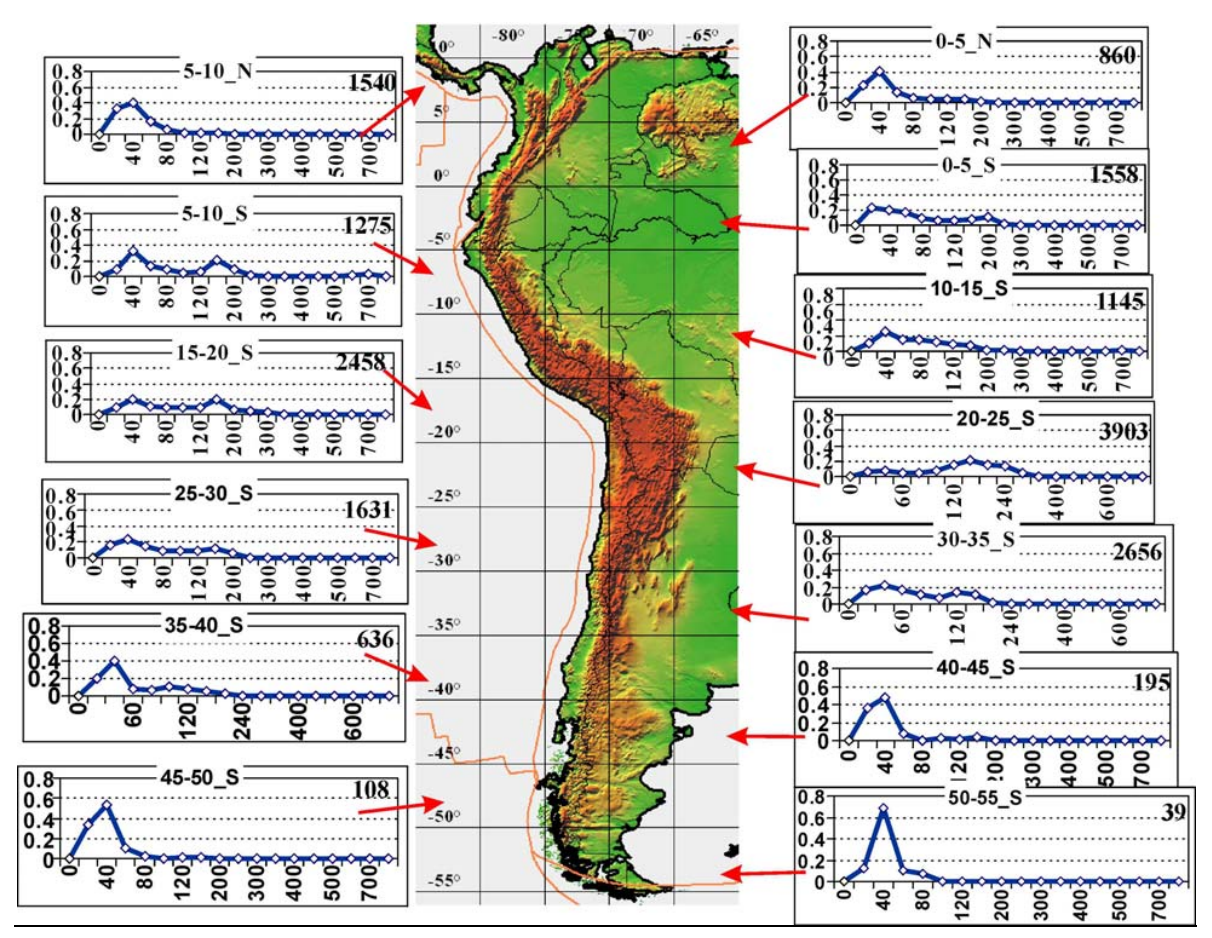

Fig. 6. The depth distributions of the EQs in each latitudinal belt (size of belt is equal to $5^{\circ}$ ) are shown in 13 plots. The vertical axes of all presented plots are relative number of the EQs in given latitudinal belt; the horizontal axes are depth in km (nonuniform scale). The red arrows indicate on the correspondent latitudinal belt. The total number of events in given latitudinal belt is shown in the upper right corner of every plot.

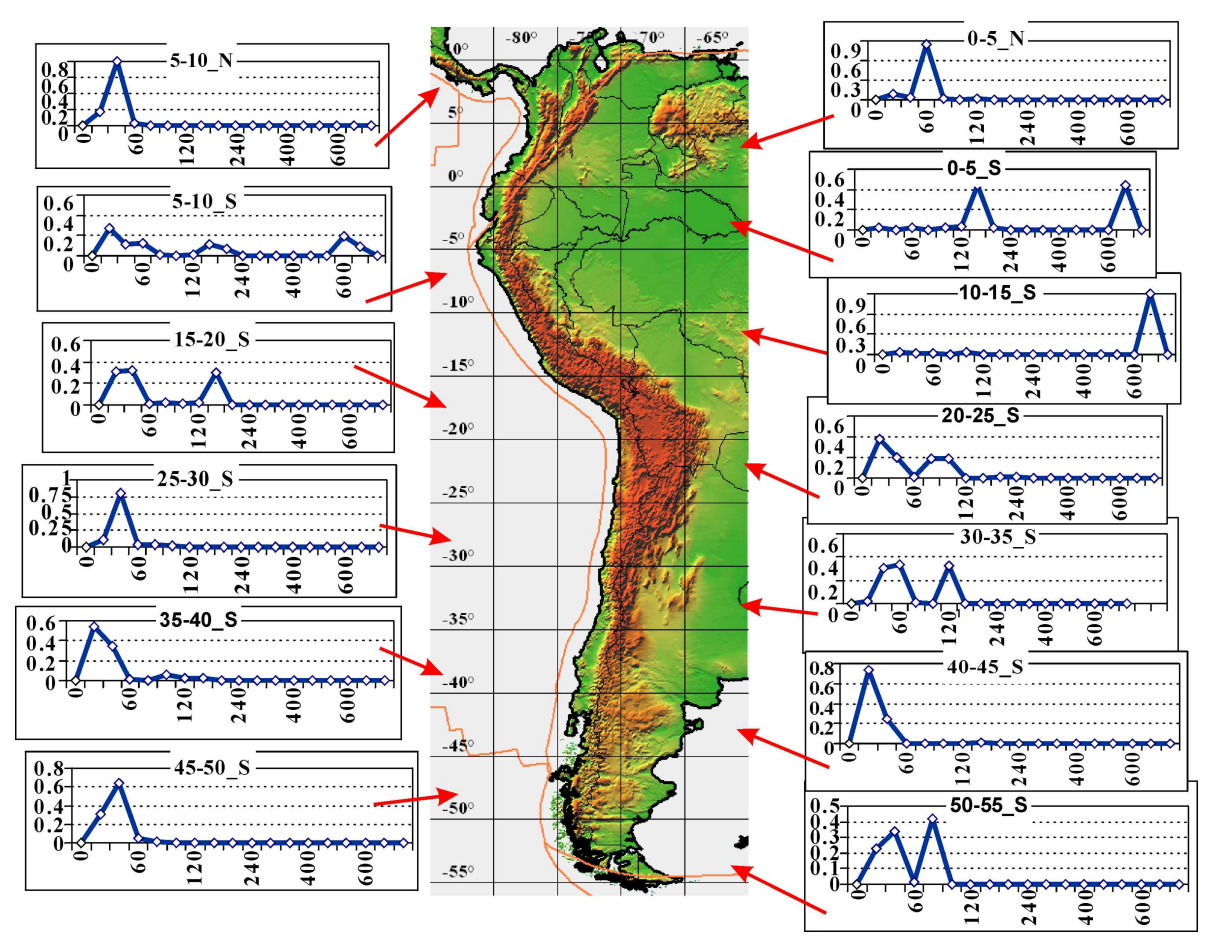

Fig. 7. The distributions of the EQ energy released in depth in each latitudinal belt (size $5^{\circ}$ ) are shown in 13 plots. The horizontal axes are depth in $\mathrm{km}$; the vertical axes are relative value of the released energy (normalization by total value of energy released in given latitudinal belt). The red arrows indicate on the correspondent latitudinal belt. 
are similar. But in the belt with local minimum $\left(25^{\circ}-30^{\circ} \mathrm{S}\right)$ between the mentioned above peaks the depths distribution is the same as in high latitudes. Sometimes in high latitudes appear bimodal distributions with peaks in depth 40 and $80 \mathrm{~km}$. It may be explained by the special depth of the EQ source (in ISC catalog) which is equal to $33 \mathrm{~km}$. Thus it is noticed general tendency as for the EQ number distributions in depth and in latitude so as for the EQ energy distributions in depth and in latitude.

\section{Discussion and conclusions}

The analysis of the obtained EQ latitudinal distributions for the Pacific part of South America shows, that the difference in the EQ number between some latitudinal belts is more than several tens times and for energy distributions this difference is more than 100 times. For example the difference in EQ number between belts $20^{\circ}-25^{\circ} \mathrm{S}$ and $40^{\circ}-45^{\circ} \mathrm{S}$ is 45 times, and the difference in energy is more than 100 times. At the same time the maximum difference in the plate traveling velocities in subduction zones in the Pacific part of the South America (from $5^{\circ} \mathrm{N}$ to $45^{\circ} \mathrm{S}$ ) is no more than $10 \%$. It seems that it difficult to explain these peculiarities of the latitudinal distributions of the EQ number and released energy only from point of view the theory of plate tectonics.

Let us discuss the possible linkage between the seismic process and tidal forces. The latitudinal distributions for whole Pacific (Fig. 1) have clearly expressed bimodal character with two peaks $\left(4^{\circ}-50^{\circ} \mathrm{N}\right.$ and $\left.20^{\circ}-30^{\circ} \mathrm{S}\right)$. It is well known from tide theory (Melchior, 1983) that the maximum of tidal energy is observed in Southern and Northern Hemispheres at the latitude $45^{\circ}$ and zero values are marked at the poles and at the equator. The same results were obtained in theoretical work (Pavlov, 2004) dedicated to the estimation of the energy accumulated in the lithosphere of the Earth due to the effects of the external and internal forces. The similarity of the latitudinal distributions of the EQ number and released energy to the tidal energy distributions is significant argument to search physical relation between these processes.

But the real latitudinal distributions for the whole Pacific shows the clearly expressed skewness, the distributions were shifted in Northern Hemisphere (peaks on $20^{\circ}-30^{\circ} \mathrm{S}$ and $40^{\circ}-50^{\circ} \mathrm{N}$ and local minimum on $10^{\circ}-20^{\circ} \mathrm{N}$ ). Now the direct relationship between the EQ origination and tidal forces is not adequately validated. It is known that significant interaction of the tidal forces on the EQ origination was noticed generally for the shallow events (Tanaka et al., 2002; Cochran et al., 2004). Moreover it was shown that weak but long-period influences of tidal forces are more effective than several times more powerful influence of short-period tidal forces (Morgounov et al., 2005, 2006). It is evidently, that composite geological medium which is characterized by nonlinear parameters and nonuniform structure, does not give simple and prompt response to an external forcing. It takes a long time for rock damage storage and energy accumulation. But the attempt to resolve this problem now is out of the frame of our work. The strongly pronounced irregularity of EQ latitudinal distributions can find the explanation in future only as a complex problem by joint analysis of the geological effects, influence of tectonic forces, tidal forces and the Earth rotation peculiarity.

The briefly, the following results have emerged from the above study:

1. The clearly expressed irregularity in latitudinal distribution of the EQ number and released energy for the Pacific part of the South America was detected. The most active seismic zone (by binormed relative number of events) according our analysis is located in the latitudinal zone $20^{\circ}-35^{\circ} \mathrm{S}$ (two peaks in belts $20^{\circ}-25^{\circ} \mathrm{S}$ and $30^{\circ}-35^{\circ} \mathrm{S}$ ). The most active energy output also occurs in the same latitudinal belts. Very remarkable variations over the depth for the EQ distribution and the energy distributions occur in studied latitudinal belts from $35^{\circ} \mathrm{S}$ to equator.

2. The sequence of calculation and statistical technique for detailed analysis of the latitudinal distributions of the EQ and two dimensional EQ distributions (in latitude and in dept) was developed.

Acknowledgements. This work was supported in part by the Russian Foundation for Basic Research (projects No. 07-05-00142, 07-05-00363, and 08-05-99098).

Edited by: B. Tilling

Reviewed by: two anonymous referees

\section{References}

Cochran, E. S., Vidale, J. E., and Tanaka, S.: Earth Tides can trigger shallow thrust fault earthquakes, Science, 306, 1164-1166, 2004.

Gutenberg, B. and Richter, C.: Seismicity of the Earth, 2-nd Edition, Princeton Univ. Press, Princeton, 217 pp., 1954.

ISC International Seismological Center: online available at: http: //www.isc.ac.uk.

Kogan, M. and Steblov, G.: Current global plate kinematics from GPS (1995-2007) with the plate-consistent reference frame, J. Geophys. Res., 113, B04416, doi:10.1029/2007/JB005353, 2008.

Melchior P.: The tides of the Planet Earth, 2nd ed., Pergamon, Oxford, 641 pp., 1983.

Mogi K.: Earthquake Prediction, Academic Press, Tokyo, 1985.

Morgounov, V. A., Boyarsky, E. A., and Stepanov, M. V.: Earthquakes and tidal phases, Izvestiya, Phys. Solid Earth, 41(1), 71$85,2005$.

Morgounov, V. A., Boyarsky, E. A., and Stepanov, M. V.: Tidal wave period and seismicity, Doklady Earth Sciences, 406(1), 112-115, 2006.

Levin, B. W. and Chirkov, Y. B.: Planetary maxima of the Earth seismicity, Phys. Chem. Earth C, 26(10-12), 781-786, 2001. 
Pavlov V. P.: Field Theory Approach to the Dynamics of a Continuous Medium: A Perturbation Theory, Theor. Math. Phys.,141(1), 1427-1442, 2004.

Levin, B. W. and Sasorova, E. V.: Bimodal Character of Latitudinal Earthquake Distributions in the Pacific Region as a Manifestation of Global Seismicity, Doklady Earth Sciences, 424(1), 175-179, 2009.
Sun, W.: Seismic energy distribution in latitude and a possible tidal stress explanation, Phys. Earth Planet. Int., 71, 205-216, 1992.

Tanaka, S., Ohtake, M., and Sato, H.: Evidence for tidal triggering of earthquakes as revealed from statistical analysis of global data, J. Geophys. Res., 107(B10), 2211, doi:10.1029/2001JB001577, 2002. 